\title{
Quantum transport properties of two-dimensional systems in disordered magnetic fields with a fixed sign
}

\author{
Tohru Kawarabayashi \\ Department of Physics, Toho University, Miyama 2-2-1, Funabashi 274-8510, Japan \\ Tomi Ohtsuki \\ Department of Physics, Sophia University, Kioi-cho 7-1, Chiyoda-ku, Tokyo 102-8554, Japan
}

(Dated: October 5, 2018)

\begin{abstract}
Quantum transport in disordered magnetic fields is investigated numerically in two-dimensional systems. In particular, the case where the mean and the fluctuation of disordered magnetic fields are of the same order is considered. It is found that in the limit of weak disorder the conductivity exhibits a qualitatively different behavior from that in the conventional random magnetic fields with zero mean. The conductivity is estimated by the equation of motion method and by the two-terminal Landauer formula. It is demonstrated that the conductance stays on the order of $e^{2} / h$ even in the weak disorder limit. The present behavior can be interpreted in terms of the Drude formula. The Shubnikov-de Haas oscillation is also observed in the weak disorder regime.
\end{abstract}

\section{INTRODUCTION}

Quantum transport in disordered magnetic fields in two dimensions(2D) has been studied widely by numerical and by analytical methods. A two-dimensional disordered system in random magnetic fields with zero mean arises in a mean field theory of the fractional quantum Hall effect at filling factor $\nu=1 / 2 . \underline{1}$ The magneto transport around $\nu=1 / 2$ has then been often analyzed by models with random magnetic fields, 1,2,3,4,5,6,7 The possibility of the Anderson transition in two-dimensional system in random magnetic fields has also been studied extensively in the last decade 8.9.10.11.12.13.14 Although much work has been done to clarify whether or not the metallic states exist in such a system which belongs to the unitary universality class, the conclusions still remain controversial. Systems in random magnetic fields show singularities at the band center in the conductance fluctuations $^{15}$ and in the density of states. ${ }^{14.16}$ These singularities are expected to be governed by the chiral symmetry of the random magnetic field system. In recent experiments, two-dimensional electron systems in random magnetic fields have been constructed and the magneto transport in such systems has been measured. ${ }^{17}$ The random magnetic fields with zero mean are realized by attaching small magnets on the layer parallel to the $2 \mathrm{D}$ electron gas in a semiconductor heterostructure. It is then found that the magneto resistance exhibits similar structure to that observed in the fractional quantum Hall effect around $\nu=1 / 2$. It is thus important to understand the transport in random magnetic fields both theoretically and experimentally.

In the present paper, we focus on another aspect of the transport in disordered magnetic fields. In the previous paper, 18 we have investigated the magneto transport in random magnetic fields and found that the conductivity is insensitive to the magnitude of the fluctuation of the random magnetic fields if the mean value $\bar{B}$ of random magnetic fields is set to be of the same order of its fluc- tuation $\delta B$. This is in contrast to the case of $\bar{B}=0$ where the conductivity diverges as $\propto(\delta B)^{-2}$ in the limit of $\delta B \rightarrow 0$. It is thus an interesting problem to clarify whether the system exhibits this insensitivity even in the limit of zero magnetic fields. We thus focus on the transport properties in the case where $\delta B \approx \bar{B}$, particularly in the limit of weak magnetic fields. The characteristic feature of such systems is that the magnetic fields have almost the same sign. We therefore consider, as a simplified model, the two-dimensional system in random magnetic fields with a fixed sign in order to analyze the case $\delta B \approx \bar{B}$.

Generally, it is expected that the scattering mechanism in random magnetic fields with a fixed sign is qualitatively different from that in the conventional random magnetic field with zero mean. The system has, for example, no snake state near the zero magnetic field lines, which plays an important role in the semi-classical theory of the transport in the conventional random magnetic fields. 6.7 It would thus be useful to study the present system also for the further understanding of the quantum transport in the conventional random magnetic fields. On the other hand, in the strong magnetic field limit $\bar{B} \gg \delta B$ it has been argued and demonstrated that the statistical properties belong to the same universality class as the quantum Hall transition in two dimensions. 19.20 The float of extended states toward the limit of the weak magnetic field $\bar{B} \ll \delta B$ has then become an important issue ${ }^{20.21}$ Clarifying the transport properties of the present case $(\bar{B} \approx \delta B)$, particularly in the limit of weak magnetic fields, would be important also in considering the connection between these two limiting cases.

We evaluate numerically the conductance in random magnetic fields with a fixed sign by the equation of motion method and by the Landauer formula. In the equation of motion method, the conductance is obtained by examining the electron diffusion directly. This method has an advantage that it applies to very large systems. On the other hand, in the case of the Landauer formula, 
large numbers of samples can be considered, although system-sizes are limited. With these two independent numerical method, we calculate the longitudinal conductivity and find that the conductance exhibits a qualitatively different behavior from that in the conventional random magnetic fields, particularly, in the limit of weak disorder. We also show that the present observations are not changed significantly by introducing a small number of magnetic fields with the opposite sign.

\section{MODEL}

We adopt a model described by the following Hamiltonian on the square lattice

$$
H=\sum_{<i, j>} V \exp \left(\mathrm{i} \theta_{i, j}\right) C_{i}^{\dagger} C_{j}
$$

where $C_{i}^{\dagger}\left(C_{i}\right)$ denotes the creation(annihilation) operator of an electron on the site $i$. The phases $\left\{\theta_{i, j}\right\}$ are related to the disordered magnetic fluxes $\left\{\phi_{i}\right\}$ through the plaquette $(i, i+\hat{x}, i+\hat{x}+\hat{y}, i+\hat{y})$ as

$$
\theta_{i, i+\hat{x}}+\theta_{i+\hat{x}, i+\hat{x}+\hat{y}}+\theta_{i+\hat{x}+\hat{y}, i+\hat{y}}+\theta_{i+\hat{y}, i}=-2 \pi \phi_{i} / \phi_{0}
$$

where $\phi_{0}=h /|e|$ stands for the unit flux and $\hat{x}(\hat{y})$ denotes the unit vector in the $x(y)$-direction. The fluxes $\left\{\phi_{i}\right\}$ are assumed to be distributed independently in each plaquette. The probability distribution $P(\phi)$ of the flux $\phi$ is given by

$$
P(\phi)= \begin{cases}1 / h_{\mathrm{rf}} & \text { for } 0 \leq \phi / \phi_{0} \leq h_{\mathrm{rf}} \\ 0 & \text { otherwise }\end{cases}
$$

The mean and the variance of the distribution are accordingly given by

$$
\left\langle\phi_{i}\right\rangle=\frac{h_{\mathrm{rf}}}{2} \phi_{0} \quad \text { and } \quad\left\langle\phi_{i} \phi_{j}\right\rangle-\left\langle\phi_{i}\right\rangle\left\langle\phi_{j}\right\rangle=\frac{h_{\mathrm{rf}}^{2}}{12} \phi_{0}^{2} \delta_{i, j}
$$

respectively.

\section{ELECTRON DIFFUSION}

In order to verify that the above system has a diffusive regime, we first examine the diffusion of electrons by means of the equation of motion method. For this, we numerically solve the time-dependent Schrödinger equation by making use of the decomposition formula for exponential operators. ${ }^{22}$ The basic formula used in the present paper is the forth order formula

$$
\exp \left(x\left[A_{1}+\cdots+A_{n}\right]\right)=S(x p)^{2} S(x(1-4 p)) S(x p)^{2}+O\left(x^{5}\right),
$$

where

$$
S(x)=\mathrm{e}^{x A_{1} / 2} \cdots \mathrm{e}^{x A_{n-1} / 2} \mathrm{e}^{x A_{n}} \mathrm{e}^{x A_{n-1} / 2} \cdots \mathrm{e}^{x A_{1} / 2} .
$$

The parameter $p$ is given by $p=\left(4-4^{1 / 3}\right)^{-1}$ and $A_{1}, \ldots, A_{n}$ are arbitrary operators. We divide the Hamiltonian into five parts as in the previous papers ${ }^{23}$ so that each part is represented as the product of $2 \times 2$ submatrices. By applying this formula to the time evolution operator $U(t) \equiv \exp (-\mathrm{i} H t / \hbar)$, we obtain

$$
U(\delta t)=U_{2}(p \delta t)^{2} U_{2}((1-4 p) \delta t) U_{2}(p \delta t)^{2}+O\left(\delta t^{5}\right)
$$

with

$$
U_{2}(x)=\mathrm{e}^{x H_{1} / 2} \cdots \mathrm{e}^{x H_{4} / 2} \mathrm{e}^{x H_{5}} \mathrm{e}^{x H_{4} / 2} \cdots \mathrm{e}^{x H_{1} / 2},
$$

where $H=H_{1}+\cdots+H_{5}$. It is to be noted that the $U_{2}$ can be expressed in an analytical form while the original evolution operator $U$ can not be evaluated exactly without performing the exact diagonalization of the whole system. This method has already been applied to the various cases of $2 \mathrm{D}^{18,23,24}$ and $3 \mathrm{D}^{25}$ disordered systems.

Numerical calculations are performed in the system of the size $999 \times 999$ with the fixed boundary condition. All the length scales are measured in units of the lattice constant $a$. To prepare the initial wave packet with energy $E$, we numerically diagonalize the subsystem $(21 \times 21)$ located at the center of the whole system and take as the initial wave function the eigenstate whose energy is closest to $E$. The single time step $\delta t$ is set to be $\delta t=0.2 \hbar / V$ and at least five realizations of random magnetic fields are considered. With this time step, the fluctuations of the expectation value of the Hamiltonian is safely neglected throughout the present simulation $(t \leq 2000 \hbar / V)$. We observe the second moment defined by

$$
\left\langle r^{2}(t)\right\rangle_{c} \equiv\left\langle r^{2}(t)\right\rangle-\langle r(t)\rangle^{2}
$$

with

$$
\left\langle r^{n}(t)\right\rangle=\int r^{n} \mathrm{~d} \Omega r^{d-1} \mathrm{~d} r|\psi(\mathbf{r}, t)|^{2}
$$

where $\psi(\mathbf{r}, t)$ denotes the wave function at time $t$. In the diffusive regime, the second moment is expected to grow in proportion to $t$

$$
\left\langle r^{2}\right\rangle_{c}=2 d D t
$$

where the diffusion coefficient is denoted by $D$ and $d$ is the dimensionality of the system. The diffusion coefficient $D$ is related to the conductivity by the Einstein relation $\sigma=e^{2} D \rho$. Here $\rho$ denotes the density of states. It is estimated by the Green function method ${ }^{26}$ for strips with the width up to 30 .

The second moment $\left\langle r^{2}\right\rangle_{c}$ for $h_{\mathrm{rf}}=0.04$ as a function of time $t$ is shown in Fig. 1. The fermi energy is assumed to be $E / V=0.5$. It is clearly seen that, above a certain length scale, the second moment grows in proportion to the time $t$. In this regime, it is natural to expect that the system is diffusive. The length scale, above which the diffusive behavior is observed, must be 


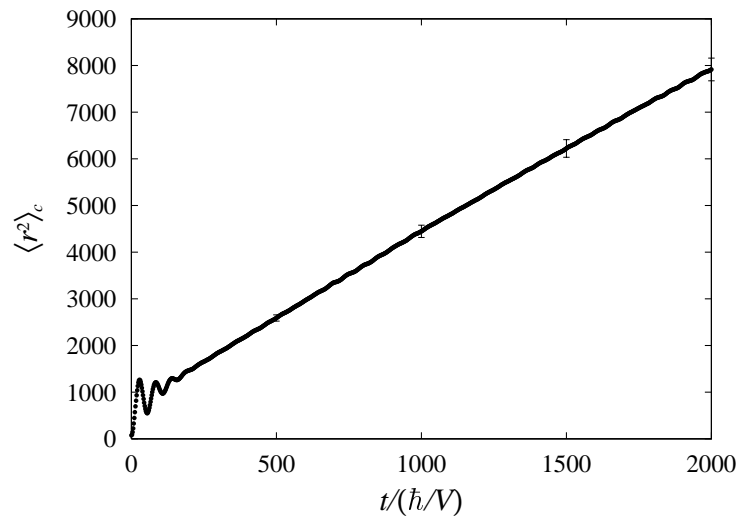

FIG. 1: The second moment averaged over 10 realization of random magnetic fields as a function of time $t$ for $E / V=0.5$ and $h_{\mathrm{rf}}=0.04$. The fluctuation around the mean value is plotted at $t /(\hbar / V)=500,1000,1500$ and 2000 .

related to the typical cyclotron radius of the present system. Since the fermi energy is fixed close to the band center, it would vary almost inversely proportional to $h_{\mathrm{rf}}$. In fact, the length scales are estimated as $\sim 38, \sim 49$, and $\sim 70$ for $h_{\mathrm{rf}}=0.04,0.03$ and 0.02 , respectively. Below these length scales, the system is in a ballistic regime. In estimating the diffusion coefficient, we discard the data in the ballistic regime. It should be noted that, in the present regime of $h_{\mathrm{rf}}$, the effective mean free path due to the fluctuation of magnetic fields is much larger than the cyclotron radius determined by the mean value of the magnetic fields. For instance, for the case of $h_{\mathrm{rf}}=0.02$, the effective mean free path is estimated to be around $2100 a, 18$ whereas the cyclotron radius is $40 a$.

The estimated conductivity is plotted as a function of $h_{\mathrm{rf}}$ in Fig. 2. It is rather remarkable that the conductivity stays on the order of the conductance quantum $e^{2} / h$ even for the small values of $h_{\mathrm{rf}}$. This feature is in contrast to the case of the conventional random magnetic fields, where the conductivity is likely to diverge inversely proportional to the square of the fluctuation of the random magnetic fields. These facts mean that, provided that the sign of the magnetic fields is fixed, the conductivity is insensitive to the strength of its fluctuation, and implies that the zero field limit $h_{\mathrm{rf}} \rightarrow 0$ does not coincide with the $h_{\mathrm{rf}}=0$ case and thus is a singular limit.

\section{CONDUCTANCE}

In order to investigate this small conductivity in the weak fluctuation regime, we examine also the conductance in the two-terminal geometry based on the Landauer formula

$$
G=\frac{e^{2}}{h} \operatorname{Tr}\left(T T^{\dagger}\right)
$$

where $T$ denotes the transmission matrix through the system. We consider the $L$ by $L$ square system with

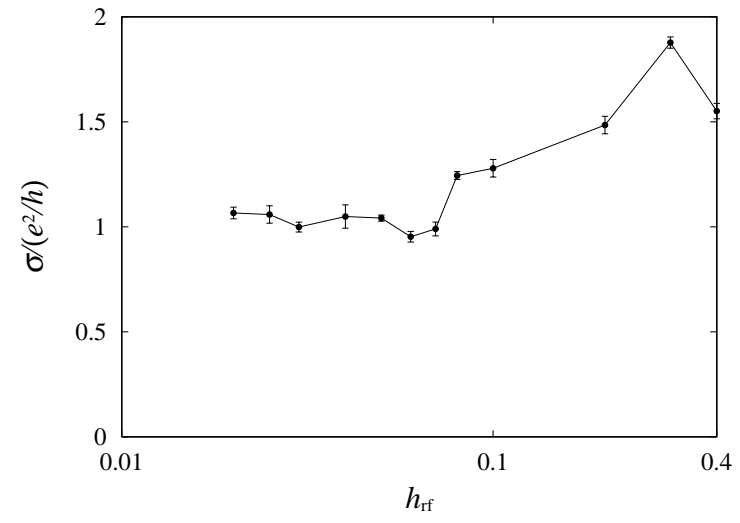

FIG. 2: The conductivity as a function of $h_{\mathrm{rf}}$ for $E / V=0.5$. For $h_{\mathrm{rf}}<0.1$, the conductivity stays on the order of the conductance quantum $e^{2} / h$.

two leads connected to both sides of the system. No magnetic field is assumed in the leads. For this system, we adopt the transfer matrix formalism ${ }^{27}$ for evaluating the transmission coefficient numerically. The maximum system-size considered in the present work is 160 by 160 and the average over 1000 realizations of random field configurations is performed. Since the mean value of the magnetic fields is not zero in the present system, the effect of the edge states is, in general, important for the transport properties. We therefore estimate the conductance both with the fixed boundary conditions and with periodic boundary conditions in the transverse direction.

In Fig. 3, the conductances for the fixed boundary conditions for various system-sizes are shown as a function of $h_{\mathrm{rf}}$. It is clearly seen that the conductance is likely to diverge as $h_{\mathrm{rf}} \rightarrow 0$, which is qualitatively different from the behavior obtained by the analysis of the electron diffusion. It is also seen that the value of the conductivity is much larger than that estimated from the electron diffusion. It is expected that these differences come from the fact that the edge states, which are absent in the simulation of the electron diffusion, exist in the case of the fixed boundary conditions. The observation that the conductance is almost inversely proportional to $h_{\mathrm{rf}}$ (Fig. 3 ) suggests that it is the manifestation of the fact that the number of edge states increases as $h_{\mathrm{rf}}^{-1}$.

In order to remove the effect of edge states, we evaluate the conductivity under the periodic boundary conditions in the transverse direction (Fig. 4). It is seen that, in contrast to the case of the fixed boundary conditions, the conductance stays on the order of the conductance quantum even for the small values of $h_{\mathrm{rf}}$. It should be emphasized that for small $h_{\mathrm{rf}}$ the conductance decreases as the size of the system is increased. We thus recover the results obtained from the electron diffusion. This suggests that it is essential to remove the effect of edge states for observing the small conductance in the random magnetic fields with a fixed sign.

In the case of the periodic boundary conditions, the 


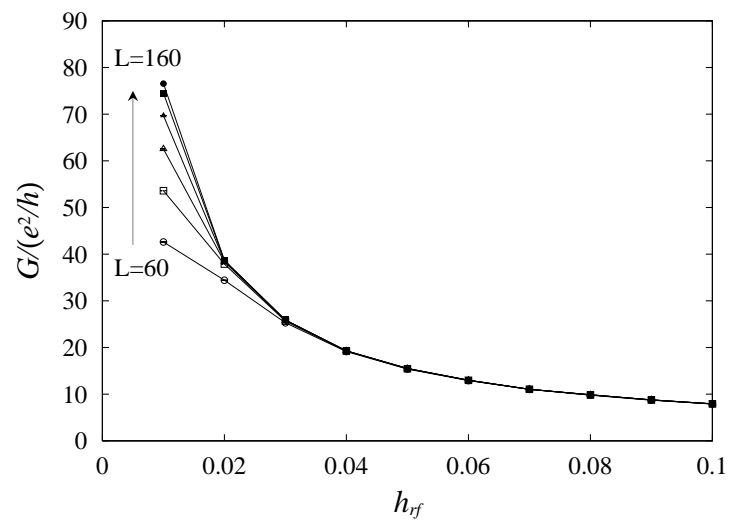

FIG. 3: The conductance under the fixed boundary conditions for $E / V=0.5$ and $L=60$ (open circles), 80(open squares), 100(open triangles), 120(solid triangles), 140(solid squares) and 160 (solid circles). The average over 1000 realizations of random fields is performed.

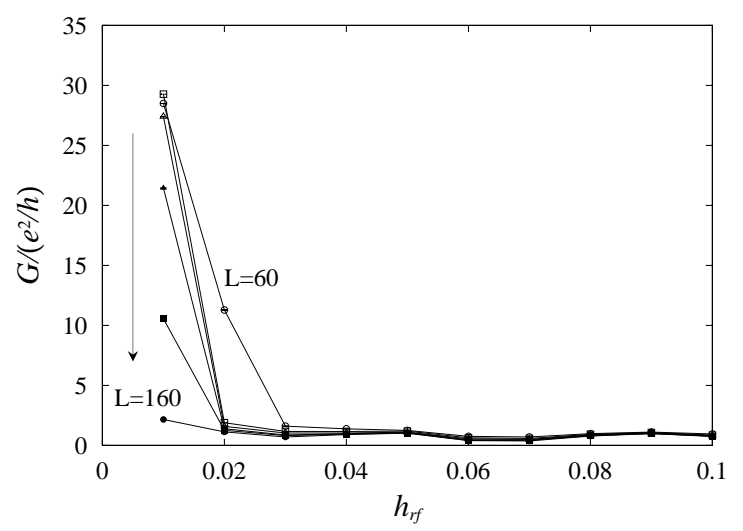

FIG. 4: The conductance under the periodic boundary conditions for $E / V=0.5$ and $L=60$ (open circles), 80(open squares), 100 (open triangles), 120(solid triangles), 140(solid squares) and 160(solid circles). The average over 1000 realizations of random fields is performed.

total flux through the system is adjusted to be an integer multiple of the flux quantum $h /|e|$ in order to ensure that the flux through the leads to be zero. Practically, we divide the excess flux into small pieces and subtract these pieces randomly from the fluxes already distributed in the system as long as they are positive, so that the above condition is satisfied. This procedure produces a weak correlation in random fluxes which should be negligible for large system sizes.

More detailed calculations of the conductance for the periodic boundary conditions are shown in Fig. 5. It is clearly seen that the conductance oscillates periodically as a function of $1 / h_{\mathrm{rf}}$ and its period is independent of the system-sizes. From our numerical data (Fig. 5), the period in $1 / h_{\mathrm{rf}}$ is estimated to be $\Delta\left(1 / h_{\mathrm{rf}}\right)=1.33$. The same type of oscillation is observed also for $E / V=0.3$ and for $E / V=0.7$ with different periods. The periods

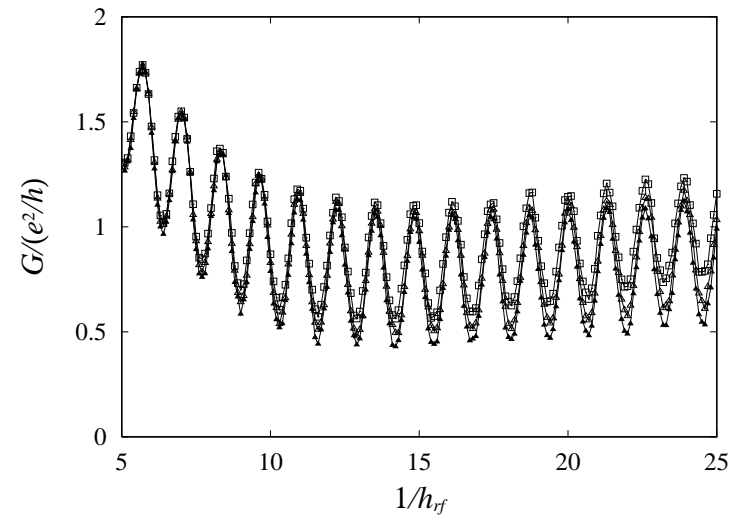

FIG. 5: The conductance under the periodic boundary conditions for $E / V=0.5$ and $L=80$ (open squares), 100 (open triangles), 120 (solid triangles) as a function of $1 / h_{\mathrm{rf}}$. The period in $1 / h_{\mathrm{rf}}$ is estimated to be 1.33 .

in $1 / h_{\mathrm{rf}}$ are estimated to be 1.18 and 1.41 , respectively. Here we show that these oscillations can be understood as the Shubnikov-de Haas effect 28 In general, the period of the Shubnikov-de Haas effect for tight-binding lattice models is given by

$$
\Delta\left(1 / \tilde{\phi}_{\mathrm{ext}}\right)=\frac{4 \pi^{2}}{a^{2} S}, \quad \tilde{\phi}_{\mathrm{ext}} \equiv \phi_{\mathrm{ext}} / \phi_{0}
$$

where $\phi_{\text {ext }}$ denotes the external flux through the plaquette and $S$ the area of the fermi surface of the twodimensional electron system. By evaluating $S$ in the system without magnetic fields, we obtain $\Delta\left(1 / \tilde{\phi}_{\text {ext }}\right)=2.36$, 2.59 and 2.83 for $E / V=0.3,0.5$ and 0.7 , respectively. Since, in the present system, the mean value of the magnetic field $\left\langle\phi / \phi_{0}\right\rangle$ is given by $h_{\mathrm{rf}} / 2$, we can identify $\tilde{\phi}_{\text {ext }}$ to be $h_{\mathrm{rf}} / 2$. We then find the relation that $\Delta\left(1 / \tilde{\phi}_{\text {ext }}\right)=2 \Delta\left(1 / h_{\mathrm{rf}}\right)$. With this relation, it is clear that the periods of the Shubnikov-de Haas effect evaluated by eq. (13) are in excellent agreement with those of the oscillation observed in our numerical data. It is rather remarkable that the present system exhibits such a clear Shubnikov-de Haas effect even though the flux distributes uniformly from 0 to $h_{\mathrm{rf}}$. A smaller oscillation than that of the conductance is observed in the density of states (Fig. 6), which seems to be consistent with the analytical results 6.29 for the conventional Shubnikov-de Haas oscillation of 2D systems in magnetic fields.

Apart from this oscillation, the conductance shows a smooth cross over from the small $h_{\mathrm{rf}}$ regime to the $h_{\mathrm{rf}}=1$ limit. It is to be noted that due to the periodicity of the phases $\theta_{i, j}$ in the hamitonian, the $h_{\mathrm{rf}}=1$ limit is identical to the case of the conventional random magnetic field with zero mean. Our numerical data suggest that this cross over takes place around $h_{\mathrm{rf}}=0.1 \sim 0.2$. With this cross over, the conductance is increased to a value $\sim 1.5 e^{2} / h$ (Fig. 5) as $h_{\mathrm{rf}}$ is increased. This enhancement of conductance in the regime $h_{\mathrm{rf}}>0.1$ is also observed in the the conductivity estimated by the equation of motion 


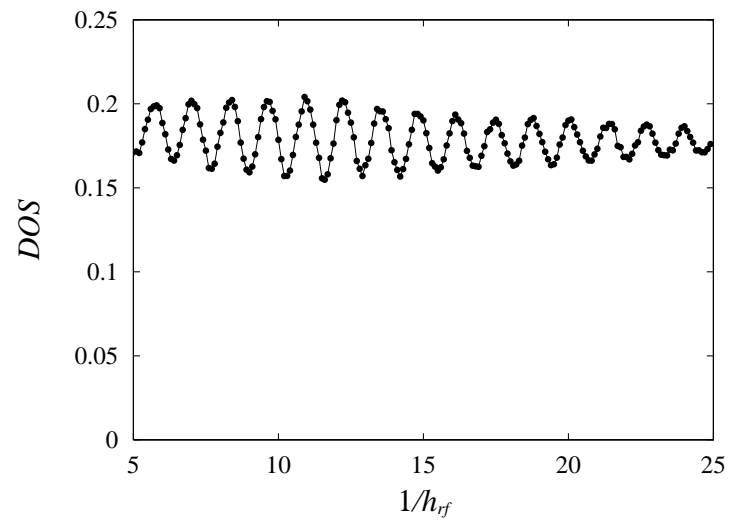

FIG. 6: The density of states for $E / V=0.5$.

method (Fig. 2).

\section{DISCUSSION}

We have investigated the longitudinal conductivity in the two-dimensional disordered magnetic fields with a fixed sign. It has been demonstrated that the system shows a slow diffusion and has accordingly a small conductance which is on the order of $e^{2} / h$ and is insensitive to the magnitude $h_{\mathrm{rf}}$ of the random fields. It would be useful to interpret these results in terms of the Drude formula $\sigma=\sigma_{0} /\left(1+\omega_{c}^{2} \tau^{2}\right)$, where $\omega_{c}$ and $\tau$ denote the cyclotron frequency and the relaxation time, respectively. The conductivity for $\omega_{c}=0$ is denoted by $\sigma_{0}$. Here it should be kept in mind that the present system is equivalent to the system in the conventional random magnetic field distributed from $-h_{\mathrm{rf}} / 2$ to $h_{\mathrm{rf}} / 2$, plus an additional uniform magnetic field $h_{\mathrm{rf}} / 2$. Namely, we can regard it as the conventional random field system in an external uniform magnetic field. From this point of view, we assume that the relaxation time $\tau$ is determined simply by the scattering due to the conventional random fields with zero mean and is insensitive to the additional uniform magnetic field. We also assume that the effect of the additional uniform magnetic field appears only in $\omega_{c}$. It is then deduced ${ }^{6.7}$ that $\sigma_{0} \propto \tau \propto h_{\mathrm{rf}}^{-2}$ and $\omega_{c} \propto h_{\mathrm{rf}}$, and therefore that for $h_{\mathrm{rf}} \ll 1$,

$$
\sigma /\left(e^{2} / h\right)=\frac{\sigma_{0} /\left(e^{2} / h\right)}{\left(1+\omega_{c}^{2} \tau^{2}\right)} \approx \frac{A_{1} h_{\mathrm{rf}}^{-2}}{\left(1+A_{2} h_{\mathrm{rf}}^{-2}\right)},
$$

where $A_{1}$ and $A_{2}$ are constants independent of $h_{\mathrm{rf}}$. If we take the limit as $h_{\mathrm{rf}} \rightarrow 0$, we obtain that $\sigma /\left(e^{2} / h\right) \rightarrow$ $A_{1} / A_{2}$. The parameters $A_{1}$ and $A_{2}$ are basically determined by the fermi energy $E \frac{6.7}{6}$ For instance, $A_{1}$ has been estimated to be 0.95 for $E / V=0.5 \stackrel{18}{t}$ The present argument based on the Drude formula, though it does not account for the Shubnikov-de Haas effect, seems to account for the fact that for $h_{\mathrm{rf}} \ll 1$ the conductance stays on the order of $e^{2} / h$ and is insensitive to $h_{\mathrm{rf}}$. In

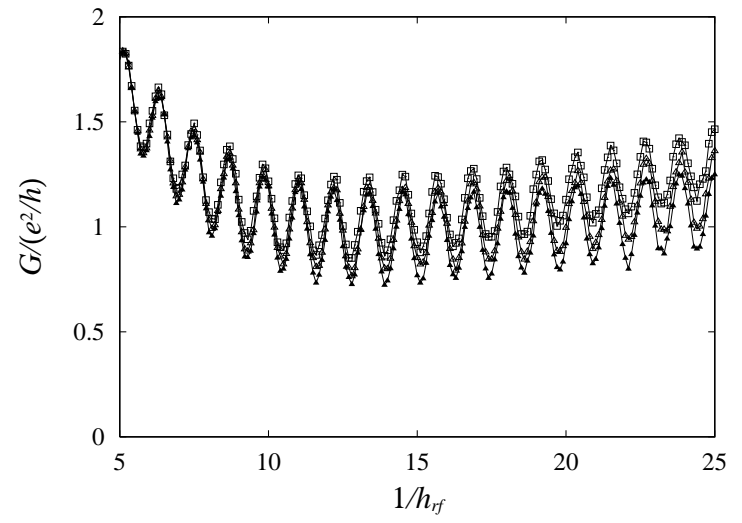

FIG. 7: The conductance for $E / V=0.5$ in the case where the random fluxes $\phi / \phi_{0}$ are distributed in the range $\left[-0.05 h_{\mathrm{rf}}, 0.95 h_{\mathrm{rf}}\right]$. Open squares, open triangles, solid triangles represent $L=80,100$ and 120, respectively.

this context, the feature specific to the present system is that both $\tau$ and $\left(\tau \omega_{c}\right)^{2}$ scales in the same way as $h_{\mathrm{rf}}^{-2}$.

Finally, we emphasize that these present transport properties are not restricted to the case of the strictly positive random magnetic fields. These properties can be observed in the case where the random magnetic fields are almost positive. In Fig. 7, the conductance is shown for the case where the random fluxes $\left\{\phi_{i} / \phi_{0}\right\}$ are distributed in the range $\left[-0.05 h_{\mathrm{rf}}, 0.95 h_{\mathrm{rf}}\right]$. It is clear that the Shubnikov-de Haas effect is also observed even though the magnetic fields are not strictly positive. This suggests that these properties are general features of systems in the random magnetic fields whose mean and fluctuation are of the same order.

In summary, we have studied numerically the transport properties in two-dimensional random magnetic fields with a fixed sign. In particular, the conductivity in the limit of small fluctuation has been investigated. In the simulation of the diffusion of electron wave functions, it is observed that the system shows diffusive behavior in larger length-scales than the typical cyclotron radius. The diffusion turns out to be very slow and the conductivity evaluated from the diffusion coefficient stays on the order of the conductance quantum insensitive to the magnitude of fluctuation of the magnetic fields. This behavior is also observed in the two-terminal conductance evaluated by means of the Landauer formula under the periodic boundary conditions. A clear Shubnikov-de Haas effect has been observed for a wide range of $h_{\mathrm{rf}}$. For a system with edge states, the contribution to the conductance from the edge channels is much larger than that from the bulk, and hence these properties are not observable. In this sense, these peculiar features are the bulk properties of the positive random magnetic fields. It has been argued that the singularity in the limit of $h_{\mathrm{rf}} \rightarrow 0$ can be understood within the framework of the Drude formula. 


\section{Acknowledgments}

The authors thank Y.Ono, B.Kramer, and S.Kettemann for valuable discussions. Numerical calculations were partly performed by the facilities of the Supercomputer Center, Institute for Solid State Physics, University of Tokyo.
1 B. I. Halperin, P. A. Lee, and N. Read, Phys. Rev. B 47, 7312 (1993).

${ }^{2}$ V. Kalmeyer and S.C. Zhang, Phys. Rev. B 46, 9889 (1992).

3 V. Kalmeyer, D. Wei, D.P. Arovas and S. Zhang, Phys. Rev. B 48, 11095 (1993).

4 A.G. Aronov, E. Altshuler, A.D. Mirlin and P. Wölfle, Phys. Rev. B 52, 4708 (1995).

5 A.D. Mirlin, D.G. Polyakov and P. Wölfle, Phys. Rev. Lett. 80, 2429 (1998).

${ }^{6}$ F. Evers, A. D. Mirlin, D. G. Polyakov, and P. Wölfle, Phys. Rev. B60, 8951 (1999).

7 A.D. Mirlin, J. Wilke, F. Evers, D.G. Polyakov and P. Wölfle, Phys. Rev. Lett. 83, 2801 (1999).

8 C. Pryor and A Zee, Phys. Rev. B 46, 3116 (1992).

9 T. Sugiyama and N. Nagaosa, Phys. Rev. Lett. 70, 1980 (1993).

10 Y. Avishai, Y. Hatsugai and M. Kohmoto, Phys. Rev. B 47, 9561 (1993).

11 A.G. Aronov, A.D. Mirlin and P. Wölfle, Phys. Rev. B49, 16609 (1994).

12 K. Yakubo and Y. Goto, Phys. Rev. B 54, 13432 (1996).

13 J.A. Vergés, Phys. Rev. B 57, 870 (1998).

14 A. Furusaki, Phys. Rev. Lett. 82, 604 (1999).

15 T. Ohtsuki, K. Slevin and Y. Ono, J. Phys. Soc. Jpn. 62, 3979 (1993)
16 C. Mudry and P. W. Brouwer, and A. Furusaki, Phys. Rev. B 59, 13221 (1999).

17 M. Ando, A. Endo, S. Katsumoto, and Y. Iye, Physica B284-288, 1900 (2000); M. Ando, Thesis (University of Tokyo, 2001).

18 T. Kawarabayashi and T. Ohtsuki, Phys. Rev. B67, 165309 (2003).

19 B. Huckestein, Phys. Rev. B53, 3650 (1996).

${ }^{20}$ K. Yakubo, Phys. Rev. B62, 16756 (2000)

21 M.C. Chang, M.F. Yang and T.M. Hong, Phys. Rev. B56, 3602 (1997).

22 M. Suzuki, Phys. Lett. A 146, 319 (1990).

23 T. Kawarabayashi and T. Ohtsuki, Phys. Rev. B51, 10897 (1995).

24 T. Kawarabayashi and T. Ohtsuki, Phys. Rev. B53, 6975 (1996)

25 T. Ohtsuki and T. Kawarabayashi, J. Phys. Soc. Jpn. 66, 314 (1997).

${ }^{26}$ L. Schweitzer, B. Kramer and A. MacKinnon, J. Phys. C17, 4111 (1984).

27 J. B. Pendry, A. MacKinnon and P. J. Roberts, Proc. R. Soc. Lond. A 437, 67 (1992).

28 C. Kittel, Quantum Theory of Solids (John Wiley \& Sons, 1987).

29 T. Ando, J. Phys. Soc. Jpn. 37, 1233 (1974). 